\title{
Intelligent system of semiotic type for decision- making support in Russia energy sector based on situational management conception
}

\author{
Liudmila V. Massel \\ Melentiev Energy Systems Institute of Siberian Branch of \\ the Russian Academy of Sciences \\ Irkutsk, Russia \\ massel@isem.sei.irk.ru
}

\author{
Aleksei G. Massel \\ the Russian Academy of Sciences \\ Irkutsk, Russia \\ amassel@gmail.com
}

Melentiev Energy Systems Institute of Siberian Branch of

\begin{abstract}
The ideas of D.A. Pospelov in situational management and semiotic modeling are proposed to interpret by means of modern information technologies. Semantic modeling is regarded as one of the areas of semiotic modeling, which is dominated by a graphical representation of developed models. It's shown the possibility to realize the general scheme of situational management using author's tools of semantic modeling, expert system and Contingency Management Language. The architecture of the Situational Polygon is proposed, which is considered as a prototype of an intelligent system of semiotic type for decision-making support in Russia energy sector based on situational management conception, including author's semantic modeling tools, contingency management language (CML) and expert system Advice.
\end{abstract}

Keywords - semiotic modeling, intelligent system, semantic modeling tools, expert system

\section{INTRODUCTION}

Intelligent decision support is becoming increasingly important in the energy sector, particularly in connection with the spread of the «Smart Power Grid» concept [1]. In this regard, of particular relevance in the energy sector is the problem of the development of intelligent control systems for complex dynamic objects. The authors propose to use to solve this problem, methods of situational management. We consider a semiotic modeling, proposed at one time DA Pospelov as a method of situational management [2]. It's suggested the mapping of Pospelov ideas on modern information technology. The semantic modeling is regarded as one of the areas of semiotic modeling, which is dominated by a graphic representation of the developed models. It's shown the possibility to implement general scheme of situational management using copyright semantic modeling tools (ontological, cognitive, event and probabilistic) and expert systems technology. The architecture of the Situation polygon is proposed as intelligent system of semiotic types for situational management in the energy sector. The task is formulated for development of expert system "Advice", included in the general scheme, and of the situational management language in extreme situations CML (Contingency Management Language) based on the author's fractal stratified approach.

\section{II . THE PROBLEM OF INTELLIGENT SYSTEMS CREATION FOR DECISION-MAKING SUPPORT IN RUSSIA ENERGY SECTOR BASED ON SITUATIONAL MANAGEMENT CONCEPTION}

At present, more and more attention is paid to the management problem of complex dynamic objects. These are modern aircraft, power plants, mobile robots, etc. As rightly pointed out in [3], they are characterized by the lack of accurate mathematical models or their excessive complexity, high dimensional state space, and the decisions made by the management, hierarchy, a variety of quality criteria, a high level of noise and external disturbances.

It is obvious that for systems with incomplete information and high complexity of the control object is becoming increasingly important application of situational management methods, based on intelligent technologies. In the energy sector, this approach is developed under the guidance of authors in the direction associated with the creation of intelligent energy systems (Smart Power Grid) [4-5].

Along with the use in intelligent energy systems of traditional methods of artificial intelligence (neural networks, genetic algorithms, fuzzy logic), more and more attention of power engineers attract so-called "goal-setting system" i.e. intelligent management systems that have multiple purposes of functioning (or are able to generate these goals), choosing the most suitable target depending on the environment, are able to predict the behavior of the environment and its own state. While such systems are lacking in energy, but it appears that the joint efforts of IT-professionals and power industry specialists aimed to developing of situational management intelligent technologies, will allow to bring a solution to this problem. The authors propose to use for solving of this problem situational management techniques based on the use of semiotic approach and semantic modeling.

\section{SituATIONAL MANAGEMENT AND SEMIOTIC MODELING BY} D.A. Pospelov.

Situational management as a scientific discipline was founded by D.A. Pospelov and was developed by him and his students and colleagues in the 80 s of the last century [2]. The need for a new approach based on the fact that there were non- 
traditional objects of management (NOM), which are called ill-defined or semi-structured, having a number of properties that distinguish them from traditional facilities of management.

The following basic properties of the objects for which it is advisable to situational management: 1) unique; 2) the lack of formalized existence purpose (control not just objects of artificial origin but conglomerates - cities, industries (including energy), ecosystems; 3) the lack of optimality ineligibility setting of the optimization task for objects of new nature; 4) dynamic - viewed objects like living systems, changes over time, their structure and function, i.e. management systems of such objects should be adaptive, willing to change his existence; 5) the incompleteness of description - usually those aspects is described that are important for a particular manager; lack of knowledge of certain aspects of the functioning of non-traditional objects, especially in disaster and emergency situations; 6) lack of a clear understanding by the technologist of the facility operation as "the most important information" can be considered only at the level of their intuition; many features of the NOM functioning, and sometimes its structure allow only qualitative, verbal description; 7) the availability of free will, or "human factor".

\section{D.A. Pospelov introduced following definitions:}

The current situation (Qj) - the set of all information about the structure of the object of control and its operation at any given time.

Complete situation (Si) - the collection consisting of the current situation, knowledge of the state management system at this moment and on the knowledge about management technology.

Logical and transformation rules (LTR) - the conversion rules of the current situation $\mathrm{Qj}$ in the current situation $\mathrm{Q} 1$ as result of control action Uk. If on-site of management object is a situation $\mathrm{Qj}$ and state of management system and process control scheme defined by $\mathrm{Si}$, allow the use of impact $\mathrm{Uk}$, then it is applied, and the current situation Qj transformed into a new situation Q1.

Classification procedures - procedures that would classify the overall situations, so that one could form as many classes as different single-step solutions is at the disposal of the control system.

Extrapolation procedures - procedures solve the choice problem of a number of possible solutions out for the full situation. They can help on the base of knowledge about the facility management and its operation previously to evaluate the results of the impact and compare the forecasts for all possible impacts in this full situation [2].

Taking into account the definitions given above, the general scheme of situational management, according to the D.A.
Pospelov includes blocks: Analyzer, Classifier, Correlator, Extrapolator and Block of random selection. They perform the following functions: Analyzer - evaluates the messages and determines the need for intervention of management system into process in the control object; Classifier - using information stored in it, considers the current situation to one or more classes that correspond to the one-step solution; Correlator - determines the LTR, which should be used. If there are several suitable LTR then Correlator enlists the aid Extrapolator that selects the best LTR out of LTR list selected by Correlator on the forecast base of possible development of situations. If a solution can not be accepted, then Block of random selection works and it's selected one of the actions that have not too much influence on the object, or the system refuses any impact. The graphical scheme will be displayed later in Fig. 2, when we will talk about displaying it on modern information technologies.

\section{SEMIOTIC MODELING}

D.A. Pospelov believed that the basis of situational management should be based on a semiotic model, which he defined as follows.

Definition 1. A formal model is called Quartet

$\mathrm{M}=<\mathrm{T}, \mathrm{R}, \mathrm{A}, \mathrm{P}>$,

where $\mathrm{T}$ - the set of basic elements, $\mathrm{R}$ - syntactic rules, $\mathrm{A}$ - the system of axioms, $\mathrm{P}$ - semantic rules.

Determination 2. A semiotic model is the model of the form

$\mathrm{C}=<\mathrm{M}, \chi \mathrm{T}, \chi \mathrm{R}, \chi \mathrm{A}, \chi \mathrm{P}>$.

where $\chi \mathrm{T}, \chi \mathrm{R}, \chi \mathrm{A}, \chi \mathrm{P}$ - respectively, the rules of $\mathrm{T}, \mathrm{R}, \mathrm{A}, \mathrm{P}$ change.

Such name of model $\mathrm{C}$ is associated with the fact that, unlike the formal models, in which the elements forming the set $\mathrm{T}$, have a hard rigid syntax, semantics and pragmatics, in model $\mathrm{C}$ all these properties of elements ti become available for modification.

But signs have exactly such feature. The signs - elements of iconic, or semiotic systems studied in semiotics [2]. Such systems are closely linked with the whole of human activity. This variability and conventionality make this activity effectively.

Sometimes the semiotic model is defined as eight:

$\mathrm{W}=<\mathrm{T}, \mathrm{R}, \mathrm{A}, \mathrm{P}, \tau, \rho, \alpha, \pi>$, where

$\mathrm{T}$ - set of main characters; R - set of syntax rules; A - a lot of knowledge about the subject area; $\mathrm{P}$ - the set of rules of inference making (pragmatic rules); $\tau$ - the rules of change of set T; $\rho$ - the rules of change of set R; $\alpha$ - the rules of change of set A; $\pi$ - the rules of change of set $\mathrm{P}[6]$.

It should be noted that the semiotic approach is developed in our country, in particular, in works of Osipov G.S. [6], Vagin 
V.N., Eremeev A.P. (intelligent real-time system of semiotic type) $[7,8]$, Kulinich A.A. (semiotic model of cognitive experience) [9], and others, but most of the work is rather theoretical. Information about the application of this approach in the field of energy could not be found.

\section{THE MODERN TREATMENT OF SITUATIONAL MANAGEMENT, AND ITS INTERPRETATION BY THE EXAMPLE OF ENERGY RESEARCH}

Decline of interest in situational management in Russia, came in 90th., besides the objective changes in the external economic and political conditions, can be explained as the ensuing of "AI winter", and the difficulties faced by developers trying to build models of complex facilities management using the proposed approach.

Nevertheless, it is now possible to ascertain a new round of interest in this area, which is reinforced by the presence of improved hardware and the appearance of new techniques and approaches, including semantic modeling. The urgency of the modern concept of situational management is also due to the fact that there was a trend called "Situation Awareness", associated with the possibility of 3D-geo_visualization of simulated situations [10]. The application of 3D-geo_visualization in the energy sector were considered by the authors, in particular, in [11]. We will use later the term "contingency management" instead of "situational management" because we have deals as usual with management in emergencies or in extreme situations in energy sector.

We use modern treatment of contingency management is used, the essence of which lies in the choice of management solutions tailored to the situation of a certain set of admissible (typical, standard) control actions [12]. Under the current situation it's understood the totality of the current state of the object (the state vector $\mathrm{X}$ ) and its external environment (vector of perturbations $\mathrm{F}$ ). Then $\mathrm{C}=<\mathrm{X}, \mathrm{F}>$.

Also the concept of complete situation is introduced:

$\mathrm{S}=<\mathrm{C}, \mathrm{G}>$, where $\mathrm{C}-$ the current situation, $\mathrm{G}-$ management goal. In turn, the control target $G$ can be represented as a target situation $\mathrm{Gg}$, which should be given the available current situation. Then $\mathrm{S}=<\mathrm{C}, \mathrm{Gg}>$.

Assuming that the current situation $C$ belongs to a class $Q$ ', and the target (given) situation $G g$ - to a class Q", a control (vector control actions $U$ ) is sought, which belongs to the set of admissible control actions $\Omega u$ and provides the required conversion of a class of situations in other :

$$
C \in Q^{\prime} \stackrel{U \in \Omega_{u}}{\longrightarrow} G_{g} \in Q^{\prime \prime}
$$

Thus, contingency management acts as a mapping:

$$
\left(Q^{\prime}, Q^{\prime \prime}\right) \rightarrow U \in \Omega_{u},
$$

assigning to a pair "the current situation - the target situation" desired result - management action $U$.

In other words, when we use the contingency management, problem of choice of control actions is reduced to an adequate assessment of the object state and the environment (which is complicated by the presence of uncertainty factors), to the attribution of the appropriate current situation to one of the standard classes and to selection of such control (from a particular set of alternatives), which leads to the achievement of the control target (the target situation ) [12] .

The above approach can be illustrated by the example of energy sector. Fig. 1 shows the general scheme of energy security (ES) problems research from the point of view of contingency management, or otherwise the estimate of the fuel energy complex (FEC) state under possible ES-threats scenarios considering measures aimed at improving the ES level. This scheme is comparable to the approach described above.

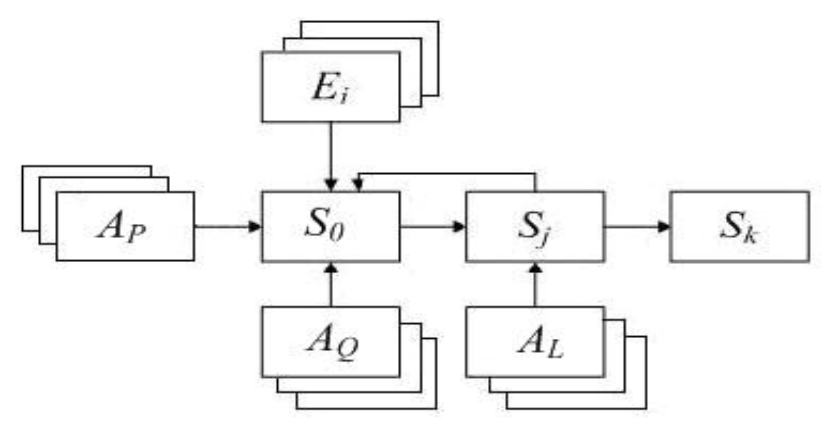

Fig. 1. General scheme of studies to assess the state Energy in terms of situational control

Here $S_{0}$ is the initial state of FEC, can be considered as the current situation $C ; E_{i}$ - scenarios of possible emergency situations in case of ES-threats realization (similar external influences $\boldsymbol{F}$ ); $A=A_{P} \cup A_{Q} \cup A_{L}$ - a set of preventive, operational and liquidation activities to prevent, neutralize or mitigate the effects of an emergency situation (can be seen as a set of related controls $\boldsymbol{U})$ ); $S_{j}$ - the FEC state after the emergency (realization of ES-threats), taking into account a set of implementation activities $A_{P}$ and / or $A_{Q} ; S_{k}$ - FEC state after liquidation measures $A_{L}\left(S_{j}\right.$ and $S_{k}$ can be seen as analogous to the corresponding target situation $\left.\boldsymbol{G}_{\boldsymbol{g}}\right)$ ).

The authors proposed to use in these studies situational analysis and situational modeling as a prerequisites for contingency management. Task of situation analysis is to identify the significant factors and parameters, or "circumstances" that define the situation, the relationships 
between them and the degree of their interference. A situation means the totality of circumstances that determine the internal state of an object or system, and circumstances which determine the state of the environment in relation to the object or system. First is described by the parameters characterizing the state of the system $(X)$, the second environmental or significant factors influencing on the development of the system $(\boldsymbol{F})$. Situational simulation is modeling of situations and of the transition from one situation to another. Situational analysis includes: analysis of problem situations (for example, emergency in energy sector); identifying of ways to resolve problem situations (alternatives) or control actions $(\boldsymbol{U})$ (in our case - the choice of the above sets $A=A_{P} \cup A_{Q} \cup A_{L}$ ); definition of criteria's for evaluating of alternatives (eg, economic ); analysis of alternatives; selection of the best alternative and analysis of its implementation consequences.

\section{THE SEMANTIC MODELING AS A MODERN INTERPRETATION OF THE SEMIOTIC APPROACH}

Given that the presence of uncertainty complicates an adequate assessment of the object and the environment states, the authors proposed to use semantic technologies of situational analysis into which the ontological, cognitive, event and probabilistic simulation are referred [15, 16]/ In fact, the semantic modeling is proposed as one of the areas of semiotic modeling, which is dominated by a graphic representation of the developed models, with elements of cognitive graphics.

Under cognitive modeling is understood to build cognitive models, or otherwise cognitive maps (directed graphs), in which vertices correspond to the factors ( concepts ) and arcs - to relations between factors (positive or negative), depending on the nature of the causal relationship [ 17]. Mathematical tools to build cognitive models is the theory of graphs.

Essence of the event simulation technique is to monitor the sequence of events on the model in the same order in which they would occur in a real system. Asked by model implementations sequence of events - a chain of events describe the scenarios of the system reaction on the occurrence of a triggering event, standing in the beginning of the chain.

Apparatus of Joiner-networks $(\mathrm{JN})$ is used as the action simulation tool. $\mathrm{JN}$ is one of the varieties of algebraic networks proposed by L.N. Stolyarov [ 18]. Joiner-network can be seen as an extension of Petri-nets, focused on behavioral modeling. The description of the interaction logic of asynchronous processes as a set of launchers and flag functions, consisting of Boolean functions, lies in the basis of JN-theory. JN peculiarity is that they provide both a graphical representation and description of a logical formulas, processing which can be automated.

Probabilistic modeling based on the use of Bayesian belief networks (BBN-modeling) and is used for simulation of energy security threats, and to assess the risks of extreme situations. More detail semantic modeling technologies and tools to support them (Intelligent IT environment) were considered by the authors in $[19,20]$

Since the results obtained by the authors, are used in a field related to energy security, situational management in this context is regarded as a contingency management (management in extreme situations in the energy sector.

Under extreme situations (ExS) in the energy sector are understood as emergency and critical situations, the determination of which is based on the assessment of the status of systems or objects on the scale of "normal", "precrisis" - a critical situation, "crisis" - an emergency. On this basis, under the critical situations defined as a situation where there are threats to the smooth functioning of the technical facilities and life support and / or threat to the life or health of individuals and social (professional) groups. These threats can be eliminated by the adoption of appropriate preventive and operational measures, which will not allow the critical situation to develop into an emergency. Currently, attention is paid mainly support decision-making and management in emergency situations where the implementation of operational and liquidation activities, it is directed, including the activities of units of Ministry of Emergency Situations. The authors give preferential attention to modeling and analysis of critical situations, allows you to create a list of preventive and operational measures to prevent possible emergencies.

Below is a mapping scheme described above situational management on the basis of the semiotic approach to semantic modeling tools developed under the guidance and with the participation of the authors in the Laboratory of Information Technologies in Institute of Energy Systems of the SB RAS (Fig. 2). 


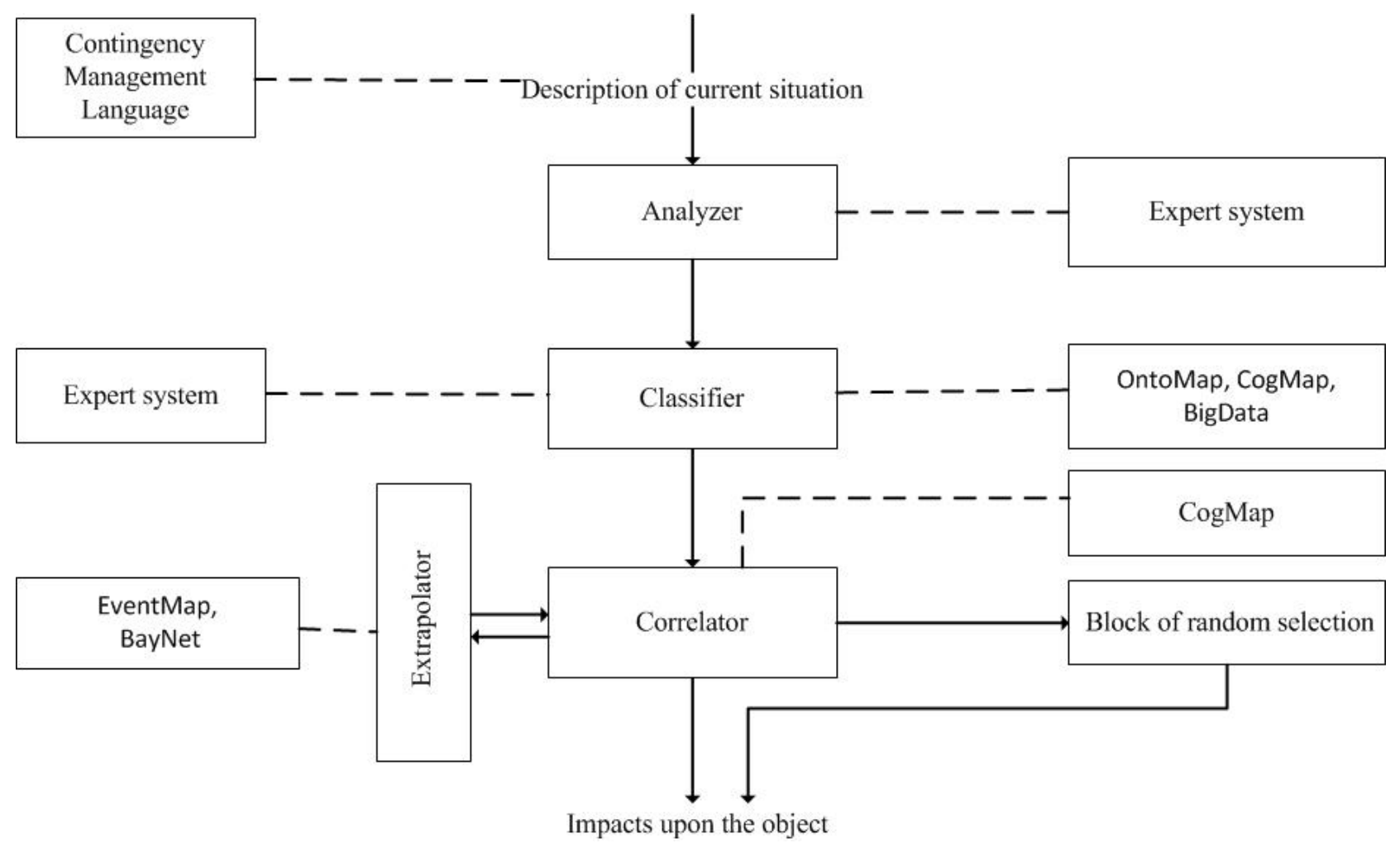

Fig. 2. Comparison of the units of the general scheme of situational management on the basis of the semiotic approach and the copyright tools of Situation Polygon.

It seems that for the performance of the Classifier functions in the intelligent system of semiotic type can be used copyrights library of ontological and cognitive modeling (OntoMap and CogMap). In addition, it may be interesting to use the concept BigData, the more that are already available tools to support it.

Extrapolator functions can perform library EventMap and BayNet (tools for event and probabilistic modeling). To analyze the situations and the choice of activities relevant to specific situations, the expert system Advice is developed.

On the basis of intelligent IT-environment under the supervision of the authors are developed two versions of the contingency management tools: Distributed Integrated Intelligent Advising System [21] and Situational Poligon. [22] The latter is considered, on the one hand, as a platform of semantic modeling, on the other, as a situation management tool.

On the Fig 3 is shown the architecture of the Situation polygon as an intelligent system of semiotic type for contingency management in the energy sector. The storage of knowledge bases and semantic models and exchange by models realize with using of the Repository approved in the framework of intelligent IT-environment. The integration of all components of the Situational polygon actualizes by using of the Contingency Management Language (CML).

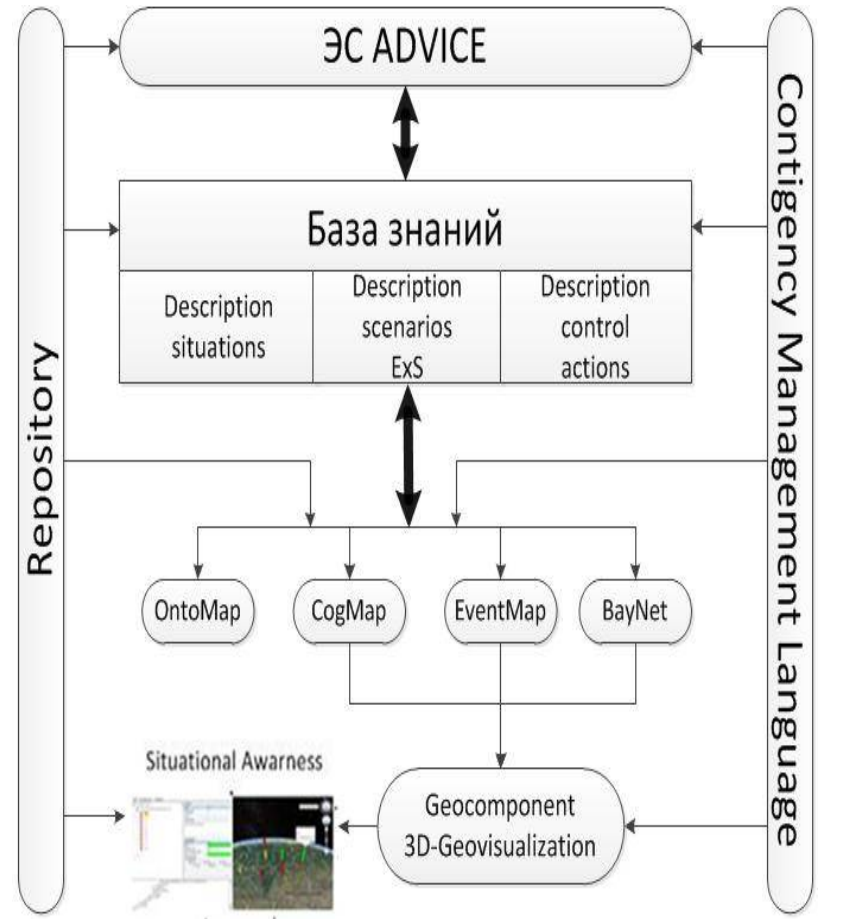

Fig. 3. The architecture of the Situation Polygon as the intelligent system of semiotic type for decision-making support in Russia energy sector based on situational management conception 


\section{DEVELOPMENT OF CONTINGENCY MANAGEMENT LANGUAGE (CML) BASED ON FRACTAL STRATIFIED MODEL}

To describe the situation and coordinate the work of the main components of the above scheme is developed CML (Contingency Management Language). For implementation is used the author fractal stratified model (FS-model), which allows to describe the information space as a set of layers including information objects of the same type, each of which, in turn, can be stratified (Fig. 4) [23].

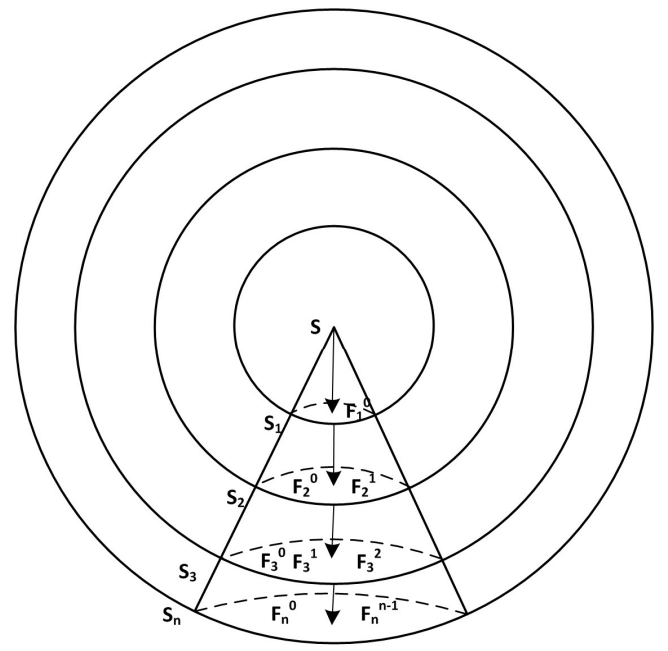

Fig. 4. Fractal stratified model. $S_{i}$ - layers (strata) of FS- model, $F_{j}^{i}$ mapping of the $\mathrm{i}$-th layer in the $\mathrm{j}$-th.

With the FS-model CML can be represented as five $\mathrm{L}=(\mathrm{C}$, $\mathrm{G}, \mathrm{E}, \mathrm{U}, \mathrm{F})$, where $\mathrm{C}$ and $\mathrm{G}$ - the description of the current and target situations, E - ExC-scenario, U - description of control actions (events), F - description of the mapping. In the corresponding FS-model can be allocated as the layers of the current and target situations $\mathrm{C}$ and $\mathrm{G}$, and as layer of ExC-scenarios E. The mapping $\mathrm{F}$ is an overlay of scenarios $\mathrm{E}$ to their current situation with generating of "transitional situation" $P$, and the impact of control actions $U$ on the "transitional situation" $\mathrm{P}$, in order to translate them into the target (stable) situation G..

Accordingly, CML should include two components (D, M): a means of the knowledge describing D (to describe situations, scenarios, control actions), and tools for knowledge manipulation $\mathrm{M}$ (for mapping of information objects from any layer in each other). These tools $\mathrm{M}$ include as a means to support the mapping $\mathrm{F}$, and, ideally, means of inference on chains (C, E, P, U. G) (the current situation C, the scenario of Exs $E$, a transitional situation $P$, control action $U$, the target situation $\mathrm{G})$.

At this stage, as a means of knowledge description (D) is proposed to use in the first step ontology of situations scenarios and events and metaontology integrating detailed ontology's. On the second and subsequent steps ontology's transform in cognitive models that reflect the causal relationships of ontology concepts, in event models describing development of the situation, and in BBN-model simulating the risks of ExS.

As a means of knowledge manipulation (M) is developed a tools to support mappings F: transition from ontology to cognitive, event and BBN models; transition from cognitive to the event models; the transition from the cognitive and event models to the BBN-models. In previous studies of authors, they were called as means of the integration of cognitive, event and BBN models. It is assumed that by means of CML will be initiated as tools of support for certain types of semantic modeling, and components providing a transition (mapping) from one type of simulation to another.

It is proposed to use CML, as shown in Fig. 3 for several purposes, such as:

1. The language for knowledge description and knowledge manipulation.

2. The tool for situations classification ("norm", critical situations, emergency situations).

3. The means of e[pert system initiation to establish a correspondence between situations and control actions.

4. Means for calling the appropriate tools of semantic modeling and mapping modules (to transit from one model type to another).

5. Means of recourse to geocomponent for 3Dgeovizualization of modeling results [24].

In other words, CML is regarded as a superstructure over the existing version of the Situation polygon [22] and executes functions as of the creation and updating of knowledge bases, and integration function of all components of the Situation polygon (Fig. 2), which, in fact, and turns it into an intelligent management system of semiotic type, because with using CML can describe rules of changes in the components of the formal model, the above: the main character sets $\mathrm{T}$; the set of syntax rules $\mathrm{R}$; the set of domain knowledge $A$; set of inference rules-making (pragmatic rules) P. A detailed description of CML is given in [25].

\section{CONCLUSION}

The article deals with the problem of creation of intelligent systems for decision-making support in Russia energy sector and proposed by the authors approach to solving them. It's described the proposed by D.A. Pospelov interpretations of situational management and semiotic modeling and display them on modern information technologies. The modern interpretation of situational management, the role and place of the semantic modeling and proposed by authors tools for support and the integration of 
different types of semantic modeling - ontological, cognitive, event and probabilistic simulations. For the first time proposed to use a semiotic approach in intelligent systems building for decision-making support in Russia energy sector. It's proposed to consider the semantic modeling as one of the areas of semiotic modeling, which is dominated by a graphic representation of the developed models, with elements of cognitive graphics. It's performed a mapping of the proposed by D.A. Pospelov general scheme of situational management on the developed tools of semantic modeling (OntoMap, CogMap, EventMap, BayNet) and Expert Systems. There is the architecture of the Situation polygon as a prototype of the intelligent system of semiotic type, based on the integration of the semantic modeling tools, expert system Advice and language for contingency management in the energy sector CML. Functions of CML are defined, on the one hand, as a means for creating and updating knowledge bases, on the other - as a tool for the integration of all components of the Situation polygon.

\section{ACKNOWLEDGMENTS}

This work was partially supported by RFBR grants №13-0700140, №15-07-1284, № 15-57-04074 and Grant Program of the Presidium of RAS №229. The authors are grateful to these organizations.

\section{REFERENCES}

[1] Kobets B.B., Volkova I.O. Innovative development of electric power based on the concept of Smart Grid / M .: IAC Energy, 2010, 208 p.

[2] Pospelov D.A. Situational management. Theory and practice. - Moscow: Nauka, 1986, 284p.

[3] Vasilyev V.I., Ilyasov B.G. Intelligent Control Systems: Theory and Practice. - M .: Radio Engineering, 2009,392 p.

[4] Massel L.V. The creation problem of intelligent and software components of Smart Grid and the approach to its solution based on the agent technology / Proceedings of XL International Conference "Information Technologies in science, education, telecommunications and business" // Application of the journal "Open Education", Ukraine, Crimea, 2012, pp. $22-25$.

[5] Massel L.V. Problems of the Smart Grid creation in Russia with a view to information and telecommunication technologies and proposed solutions / The 15th International Workshop on Computer Science and Information Technologies (CSIT'2013). - Hungarian Academy of Sciences, Budapest, Hungary, 2013, Vol. 1, pp. $115-120$.

[6] Osipov G.S. From situational management to applied semiotics / News of artificial intellegence. 2002, №6 (54), pp. 2-12.

[7] Vagin V.N., Eremeev A.P. Some basic principles of intelligent decision support systems for the real-time // News of Russian Academy of Sciences. Control Theory and System. 2001, №6, pp. 114-123

[8] Eremeev A.P. Application of reinforcement learning in intelligent real-time systems / Proceedings of Congress on Intelligent Systems and Information Technology «IS \& IT'14». - M .: Fizmatlit, 2014, V. 1, pp. 263-271.

[9] Kulinich A.A. Semiotic cognitive architecture of DSS / Proceedings of Congress on Intelligent Systems and Information Technology «IS \& IT'14». - M .: Fizmatlit, 2014, V.2, pp. 146-153.
[10] Endsley, M. R. The Role of Situation Awareness in Naturalistic Decision Making. In C. Zambock \& G. Klein (Eds.), Naturalistic Decision Making, Mahwah, NJ: LEA. 1997, pp. 269-284.

[11] Massel L.V., Ivanov R.A., Massel A.G. Simulation of decision-making stages based on network-centric approach / Vestnik of ISTU. № 10 (81), 2013, pp. 16-22.

[12] Vasilyev V.I. Intelligent information security system - Mashinostroyeniye, 2012, $171 \mathrm{P}$.

[13] Massel L.V., Massel A.G. Contingency management and semantic modeling in the energy sector / Proceedings of the IV International Conference OSTIS, Belarus, Minsk, BSUIR. 2014, pp. 111-116.

[14] Massel L.V., Massel A.G. Methods and tools for contingency management in the energy sector on the basis of semantic modeling / Proceedings of the V International Conference OSTIS, Belarus, Minsk BSUIR. - 2015, pp. 199-204.

[15] Massel L.V. Application of ontological, cognitive and event simulation to analyze the development and consequences of emergencies in Energy / Security and emergencies. - № 2, 2010, pp. 34-43.

[16] Massel L.V., Massel A.G. Semantic technologies are based on the integration of the ontological, cognitive and event simulation / Proceedings of the III International Scientific and Technical Conference «OSTIS-2013" - Belarus, Minsk: BSUIR, 2013, pp. 247-250.

[17] Trahtengerts E.A. Computer support decision-making. - M.: SINTEG, 1998, 376 P.

[18] Stolyarov L.N. Philosophy event simulation as an example scenario energy catastrophe / / Proceedings of the International Conference "Information Technologies in science, education, telecommunications and business." Ukraine, Gursuf 2010, pp. 197-200.

[19] Massel L.V., Massel A.G. Intelligent computing in research of directions development in energy sector / / Bulletin of the Tomsk Polytechnic University. Management, Computer Science and Informatics. - 2012, V. 321, № 5, pp. 135-141.

[20] Massel A.G. Intelligent IT-environment for research of energy security / Proceedings of the International Conference "Information Technologies in science, education, telecommunications, and business." Supplement to the journal "Open Education". - Ukraine, Gurzuf, 2010, pp. 306-309.

[21] Massel L.V., Massel A.G. Distributed intelligent advising system to decision making support in extreme situations / Proceedings of XIL International Conference "Information Technologies in science, education, telecommunications, and business." - Ukraine, Crimea. - Zaporozhye: Zaporozhye State University, 2013, pp. 145-149.

[22] Massel A.G., Ivanov R.A. Situational polygon as tools of contingency management in the energy sector/ Proceedings of the IV International Conference OSTIS. - Belarus, Minsk BSUIR. - 2014, pp 277-280.

[23] Massel L.V. Fractal model of knowledge structuring / Proceedings of the National conferences, with international participation "Artificial Intelligence-94" .- Rybinsk, 1994. V.1, pp. 46-49.

[24] Massel L.V. Fractal approach to the construction of information technologies / In the book: Krivorutsky L.D., Massel L.V. // Information Technology of Energy Development Research // Novosibirsk: "Science", Ed. company RAS, 1995, pp 40-67.

[25] Massel L.V., Massel A.G .The language of knowledge description and knowledge management in the intelligent system of semiotic type / Proceedings of the XX Baikal Russia conference "Information and mathematical technologies in science and management." Part III.- Irkutsk: ESI SB RAS, 2015, pp. 112-124. 